\title{
Optimizing Stroke Care for Patients with Large Vessel Occlusions: Current State of the Art and Future Directions
}

Johanna M. Ospel, ${ }^{1,2}$ Arnuv Mayank, ${ }^{1}$ Shinichi Yoshimura, ${ }^{3}$ and Mayank Goyal ${ }^{4}$

Acute ischemic stroke (AIS) is a severely disabling disease. Endovascular therapy is a powerful and highly effective treatment option for these patients and has recently become standard of care. The benefits of endovascular treatment (EVT) are tremendous both from a patient and from an economic perspective, since it dramatically improves individual patient outcomes while reducing long-term healthcare costs at the same time. The effect of EVT is highly time-dependent. Thus, the overarching goal in AIS is to quickly transport and diagnose the patient to minimize treatment delays. In this review, we provide an overview about the current state of stroke care, propose a fast and simplified imaging protocol and management approach for AIS patients. We also highlight the challenges we are currently facing in endovascular stroke treatment and suggest possible solutions to overcome these.

Keywords $>$ acute ischemic stroke, endovascular therapy, mechanical thrombectomy, stroke imaging, multiphase CT angiography

\section{Background: Natural History of Acute Ischemic Stroke}

Acute ischemic stroke (AIS) is a severely disabling disease, particularly when there is an underlying large vessel occlusion (LVO). Until recently, intravenous alteplase was the only available treatment option for LVO patients, but its efficacy was limited, as recanalization of the occluded vessel was only achieved in $7 \%-30 \%$ of patients, ${ }^{1)}$ and many patients were not eligible for alteplase treatment at all because of the numerous contraindications and narrow

${ }^{1}$ Department of Clinical Neurosciences, University of Calgary, Calgary, Canada

${ }^{2}$ Division of Neuroradiology, Clinic of Radiology and Nuclear Medicine, University Hospital Basel, University of Basel, Basel, Switzerland

${ }^{3}$ Department of Neurosurgery Hyogo College of Medicine, Nishinomiya, Hyogo, Japan

${ }^{4}$ Department of Radiology, University of Calgary, Calgary, Canada

Received: February 12, 2020; Accepted: March 4, 2020

Corresponding author: Mayank Goyal. Departments of Radiology and Clinical Neurosciences, Foothills Medical Centre, 1403 29th St. NW, Calgary, AB, T2N2T9, Canada

Email:mgoyal@ucalgary.ca

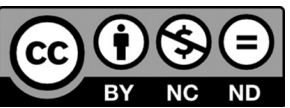

This work is licensed under a Creative Commons Attribution-NonCommercialNoDerivatives International License.

(C)2020 The Japanese Society for Neuroendovascular Therapy therapeutic window (within 4.5 hours from symptom onset). ${ }^{2}$ It is estimated that only $3 \%-5 \%$ of AIS patients ultimately receive intravenous alteplase. ${ }^{3)}$ Clearly, there was a need for another alternative treatment strategy to reduce the morbidity and mortality in AIS. Therefore, endovascular treatment techniques (EVT), which rely on mechanical clot retrieval rather than pharmacological recanalization, have been developed. In 2015, ischemic stroke treatment has fundamentally changed. Five major randomized controlled trials have proven the superiority of EVT compared to medical management in patients with LVO strokes. ${ }^{4)}$ EVT is now considered standard of care for these patients. ${ }^{2}$

\section{Why Offering Endovascular Therapy for AIS?}

\section{Benefit from a patient perspective}

There are several reasons why EVT should be performed for LVO strokes. First and foremost, the benefit of EVT compared to best medical care from a patient perspective is overwhelming: The number needed to treat for reduction of disability by at least one point on the modified Rankin Scale (mRS) is 2.6, one of the lowest throughout the history of medicine. ${ }^{4)}$ This translates into a substantial increase in quality-adjusted life years (QALYs). ${ }^{5}$ )

The safety profile of EVT is excellent, with no significant differences in mortality and symptomatic intracranial 
Table 1 Established patient transport paradigms in acute ischemic stroke

\begin{tabular}{|c|c|c|c|}
\hline $\begin{array}{l}\text { Transport } \\
\text { paradigm }\end{array}$ & Description & Advantages & Disadvantages \\
\hline $\begin{array}{l}\text { Direct to } \\
\text { mothership }\end{array}$ & $\begin{array}{l}\text { The patient is directly transported } \\
\text { to the nearest CSC, bypassing } \\
\text { PSCs even if they are closer than } \\
\text { the CSC }\end{array}$ & $\begin{array}{l}\text { Since every patient gets } \\
\text { admitted to an EVT-capable } \\
\text { center, EVT treatment } \\
\text { delays are minimized }\end{array}$ & $\begin{array}{l}\text { Time to intravenous alteplase treat- } \\
\text { ment might be longer when PSCs are } \\
\text { bypassed } \\
\text { Risk of overwhelming CSCs with } \\
\text { non-LVO strokes } \\
\text { PSCs lose their patients which can } \\
\text { lead to loss of revenue, jobs, etc. }\end{array}$ \\
\hline Drip and ship & $\begin{array}{l}\text { The patient is transported to the } \\
\text { nearest PSC for intravenous } \\
\text { alteplase treatment and in case an } \\
\text { LVO is present, he/she is trans- } \\
\text { ferred to a CSC }\end{array}$ & $\begin{array}{l}\text { Time to intravenous } \\
\text { alteplase is minimized as } \\
\text { transport times to the PSC } \\
\text { is usually shorter than to the } \\
\text { nearest CSC }\end{array}$ & $\begin{array}{l}\text { EVT is delayed in LVO patients since } \\
\text { they have to be redirected from the } \\
\text { PSC to the CSC after the LVO diagno- } \\
\text { sis has been confirmed }\end{array}$ \\
\hline Mobile stroke unit & $\begin{array}{l}\text { A CT-equipped specialized } \\
\text { ambulance is dispatched to the } \\
\text { site of the stroke and after } \\
\text { intracranial hemorrhage has been } \\
\text { ruled out on CT, treatment with } \\
\text { intravenous alteplase is initiated }\end{array}$ & $\begin{array}{l}\text { Intravenous alteplase can be } \\
\text { initiated in the pre-hospital } \\
\text { setting, which can minimize } \\
\text { alteplase treatment delays in } \\
\text { certain geographies }\end{array}$ & $\begin{array}{l}\text { High initial outlays and maintenance } \\
\text { costs } \\
\text { Depending on the geography, number } \\
\text { and location of available MSUs, the } \\
\text { time to reach the patient can be long }\end{array}$ \\
\hline
\end{tabular}

EVT: endovascular treatment

hemorrhage compared to medical management. ${ }^{4)}$ Thus, many physicians, including ourselves, now offer EVT routinely beyond guideline recommendations. ${ }^{6,7)}$ This is in accordance with patients' preferences, who also prefer more aggressive treatment approaches. ${ }^{8)}$

\section{Benefit from a societal perspective}

In medicine, there are many therapies that benefit the individual but are so expensive that it becomes almost impossible for doctors to balance their duties toward patients and the society. ${ }^{9)}$ For EVT, the situation is different, as its cost-effectiveness has been proven in several industrial nations and developing countries. ${ }^{10-13)}$ It is true that the initial expenses are higher with EVT, due to the price of catheters and stent retrievers, and because of the resources that are needed to establish a comprehensive network of EVT centers. But in the long run, reductions in morbidity and mortality outweigh these initial expenses.

\section{How Should EVT Be Performed?}

In AIS, neurons die at a rapid pace. The average AIS patient loses 1.9 million neurons per minute, ${ }^{14)}$ and every 30 -minute delay in recanalization decreases the chance of a good functional outcome by $8 \%-14 \% .{ }^{15)}$ Reperfusion quality, that is, how well the vessel occlusion is reopened, is a key determinant of patient outcome: higher expanded Thrombolysis in Cerebral Infarction (eTICI) grades are strongly associated with improved patient outcomes. ${ }^{16)}$ Reperfusion quality deteriorates with increasing time to treatment, probably because of changes in clot composition. ${ }^{17)}$ Firstpass effect (i.e., achieving complete revascularization with a single device pass) is an independent predictor of good outcome, as multiple device passes are associated with reperfusion delays and an increased risk of vessel injury. ${ }^{18)}$ Thus, fast and complete first-pass reperfusion should be the goal when performing EVT, not only for the sake of a better functional outcome but also to minimize healthcare costs: the net monetary benefit for an average US patient is approximately $\$ 17000$ per $1 \%$ increase in the final eTICI $2 \mathrm{c} / 3$ rate and $\$ 10600$ per 10 minutes time to treatment decrease. ${ }^{5,19)}$

In the following, we outline the challenges that are encountered in the pre-hospital and in-hospital phase when performing EVT in AIS patients and suggest possible strategies for to overcome these problems. 


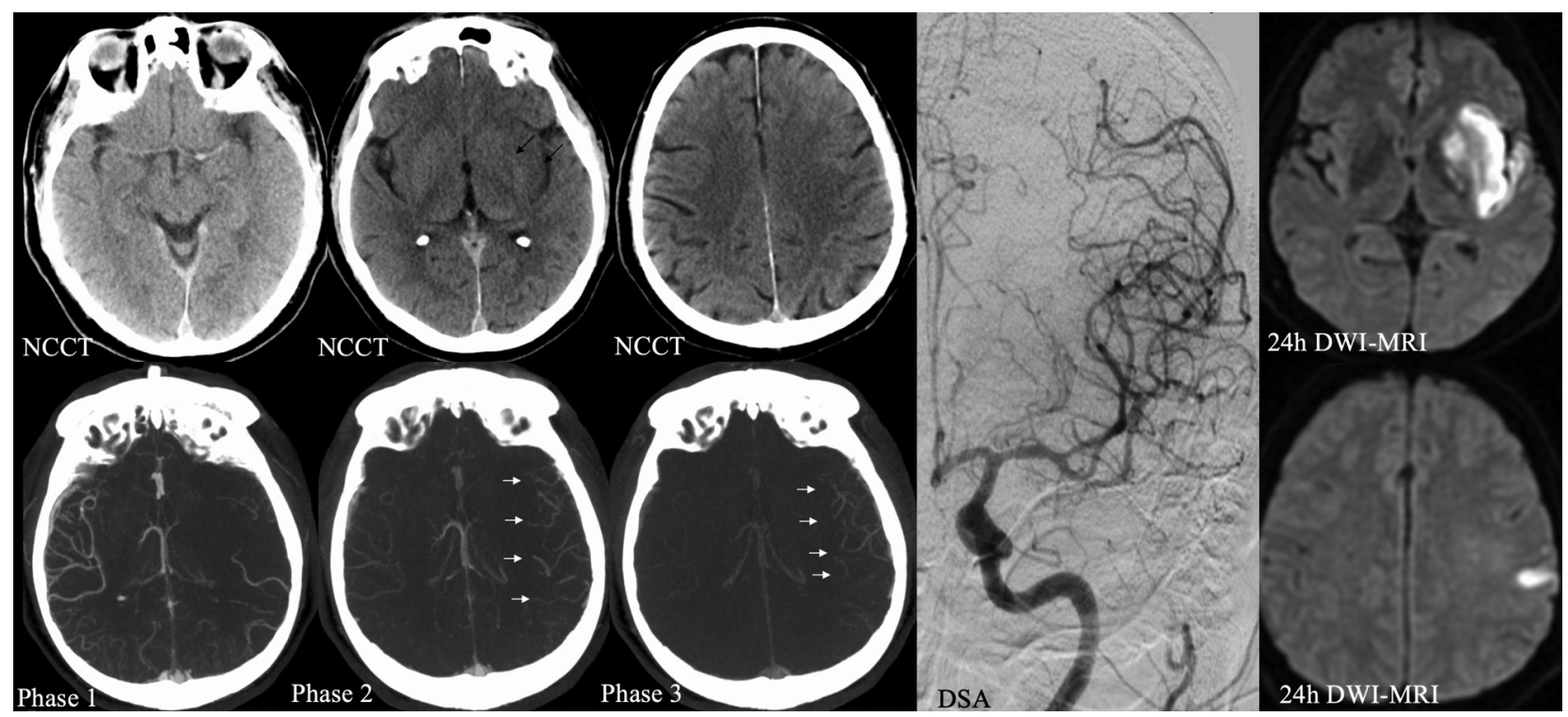

Fig. 1 Acute stroke imaging protocol. First, a NCCT is obtained to rule out intracranial hemorrhage and provide a rough estimate about ischemic core. Here, it reveals a hyperdense vessel sign of the left middle cerebral artery suspicious for a left M1 occlusion, hypodensity and loss of grey white matter differentiation of the left insula and lentiform nucleus (ASPECTS of 8, black arrows). Multiphase CT angiography is obtained immediately afterwards. The first phase covers the intra- and extracranial vasculature from skull base to vertex, and provides additional information about the anatomy of cervical vessels that can be used to choose appropriate catheters and devices. The second and third phase cover the intracranial vasculature from skull base to vertex and show moderate to good collateral opacification with good extent and predominantly a one-phase delay in collateral filling (white arrows). Taken together, these imaging findings strongly speak in favor of EVT. The patient was treated with intravenous alteplase and EVT, and fast eTICI 3 reperfusion could be achieved (see final DSA run). Follow-up diffusion-weighted MRI revealed an infarct core that matched the initial changes on NCCT with almost no additionally infarcted tissue. ASPECTS: Alberta Stroke Program Early CT Score; eTICl: expanded Treatment in Cerebral Infarction; EVT: endovascular treatment; MRI: magnetic resonance imaging; NCCT: non-contrast CT

\section{What Are the Challenges in the Pre-hospital Phase?}

\section{Accurate pre-hospital LVO detection}

Currently, it is not possible to detect LVOs in the pre-hospital phase. Patients have to be transferred to a hospital, where CT angiography (CTA) is then performed to confirm or rule out an LVO. Most hospitals are capable to perform CTA but not all of them provide EVT. Thus, many LVO patients are initially transferred to a non-capable EVT center, which can result in substantial treatment delays and worse patient outcomes. ${ }^{20)}$ A tool that allows for pre-hospital LVO detection is urgently needed, but not available yet. Clinical severity scores are unable to reliably distinguish between LVO and non-LVO strokes. ${ }^{21)}$ However, several promising technologies such as ultrasound-based LVO detection in the ambulance ${ }^{22)}$ are currently in development and could soon facilitate pre-hospital patient triage and transport decisions.

\section{Patient transport}

EVT is now considered standard of care for AIS patients, but, as is often the case, clinical reality is lagging behind guideline recommendations, and in many countries, particularly in the developing world, EVT is not available at all. In other areas, even in industrial nations, comprehensive networks of EVT centers are not established yet, as the equipment and expertise needed to provide EVT is mainly clustered in large cities, while in rural areas, access to EVT is still lacking. Thus, ambulances often have to cover long distances to transport an LVO patient to the nearest comprehensive stroke center (CSC). This is complicated by the fact that besides CSCs that offer EVT 24/7, there are many smaller hospitals, the so-called primary stroke centers (PSCs), which only offer intravenous alteplase treatment, but not EVT. If an LVO patient is initially transferred to a PSC, and then re-directed to a CSC after an LVO has been confirmed, this leads to delays in treatment and worsening of outcomes. ${ }^{23)}$ But although the chances of an LVO opening with intravenous alteplase alone are small, this possibility cannot be completely disregarded. Thus, universal bypass to a CSC may not always be appropriate, and it could also lead to an overwhelming workload at the CSC, with many non-LVO strokes being admitted that could also be treated at the PSC. Several different transport paradigms are currently in use (Table $\mathbf{1}$ ). 


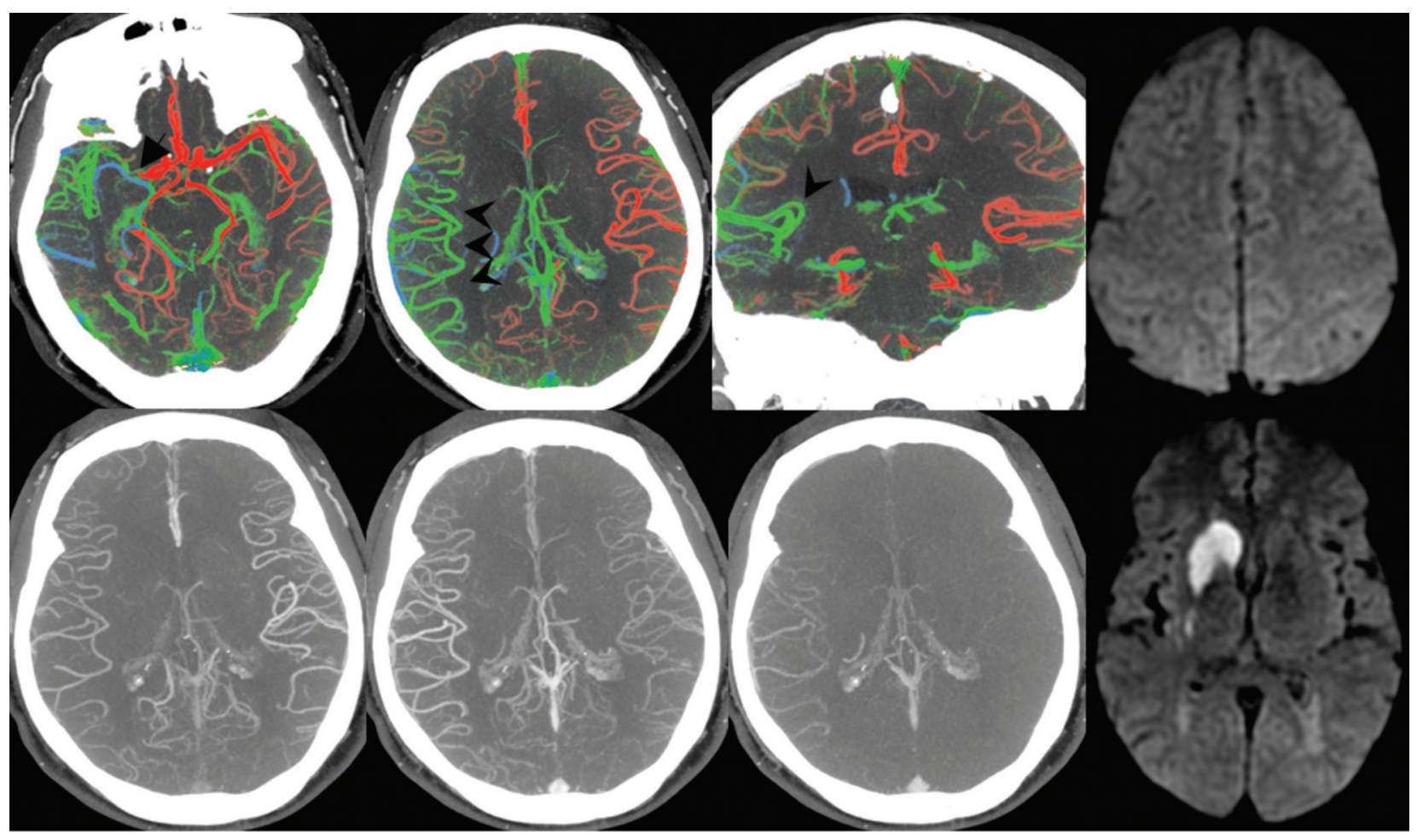

Arterial phase

Peak venous phase

Late venous phase

Fig. 2 Right-sided M1 segment MCA occlusion (arrow). On color-coded MCTA summation maps (top row), the vessels in the affected hemisphere are predominantly green, which indicates a one-phase delay. The number of vessels is similar to the contralateral side. Taken together, these findings suggest excellent collateral filling. Regular MCTA images are can be seen in the bottom row. Diffusion-weighted imaging was obtained on the next day and revealed only a small infarct in the caudate head and lentiform nucleus. CT: computed tomography; mCTA: multiphase computed tomography angiography (Reprinted with permission from Displaying Multiphase CT Angiography Using a Time-Variant Color Map: Practical Considerations and Potential Applications in Patients with Acute Stroke. J.M. Ospel, O. Volny, W. Qiu, M. Najm, N. Kashani, M. Goyal, B.K. Menon. American Journal of Neuroradiology Jan 2020)

There is no one-size-fits-all solution for stroke patient transport. Which transport paradigm performs best is highly context-specific and depends on several factors:

1) the likelihood of an LVO in a given patient;

2) local geography;

3) workflow efficacy at the local PSCs and CSCs;

4) available transport resources (number of ambulances, air transport capacities, etc.).

Conditional probability modelling can be used to predict which transport paradigm might be the most suitable, as it is able to synergize information about local geography, available transport resources, and workflow efficiency of the local hospitals..$^{24,25}$ Broadly speaking, these models show that in systems with high workflow efficiency and small geographies, the mothership model is often most effective in reducing transport times, although performance of both paradigms can be very similar. In larger geographies, the drip-and-ship model can sometimes be beneficial. The data regarding MSU and the flying/driving neurointerventionist approach are still limited at the current moment. ${ }^{26,27)}$ It is important to note that streamlining of in-hospital workflows is important, but it is pre-hospital patient triage and transport paradigms which yield the greatest potential for time savings. ${ }^{28-30)}$

\section{Effective communication and pre-notification of the medical team}

Pre-notification of the medical team, and particularly the neurointerventionalist, for example, through a phone call or an app allows the medical team to set up the neuroangiography suite for EVT even before the patient arrives and can very effectively reduce treatment delays. ${ }^{31)}$ However, pre-notification is not routinely used yet, ${ }^{32)}$ partly because of the high costs associated with it. It would be desirable for industry to develop affordable but yet intuitive and easy-to-use communication tools that allow for broad utilization of pre-notification. 


\section{What Are the Challenges in the In-hospital Phase?}

\section{Imaging paradigm}

Imaging is an essential part of AIS management as it establishes the diagnosis, confirms the presence of an LVO, and thereby guides further treatment. The two questions that have to be answered by acute stroke neuroimaging are ${ }^{33)}$ :

1) Is there intracranial hemorrhage?

2) Is there a vessel occlusion and where is it located?

Ideally, acute stroke imaging should be readily available, easy to interpret, fast, and inexpensive. Due to the several drawbacks of MRI (numerous contraindications, limited availability, longer scan times, and susceptibility to patient motion), most centers, including our own, rely on CT imaging in the acute stroke setting.

Non-contrast CT (NCCT) is typically used to differentiate between hemorrhagic and ischemic stroke. Hemorrhagic stroke is characterized by hyperdense hemorrhagic foci that can easily be detected even by physicians with limited imaging experience. NCCT also provides us with a rough estimate of the extent of early ischemic changes. Ischemic regions appear hypodense on NCCT, and the Alberta Stroke Program Early CT Score (ASPECTS) is a semi-quantitative score that can be used to assess the extent of hypoattenuation in MCA strokes. ${ }^{34)}$ Low ASPECTS scores indicate a greater degree of ischemic damage and are associated with higher risks of intracranial hemorrhage and worse outcomes, with and without treatment. Having said that, one should be cautious not to over-rely on imaging findings when deciding about treatment, as even patients with very low ASPECTS scores can still benefit from EVT. ${ }^{35}$

Once intracranial hemorrhage has been ruled out and the extent of early ischemic changes has been roughly estimated on NCCT, one has to determine whether there is a possible target lesion for EVT (LVO or EVT-accessible medium vessel occlusion). This requires vascular imaging. In our opinion, multiphase CTA (mCTA) should be the vascular imaging method of choice, since it allows physicians to quickly and reliably detect large and medium vessel occlusions while providing additional information about collateral supply at the same time. This is important, since good collaterals are independently associated with improved functional outcome after EVT. ${ }^{36)}$ mCTA predicts outcome more accurately than single phase CTA and CT perfusion (CTP $)^{37)}$ is robust against patient motion, requires no post- processing, and covers the whole brain. In our opinion, the optimal imaging protocol consists of a non-contrast head CT to rule out intracranial hemorrhage and provide an estimate of ischemic changes, followed by mCTA to confirm the presence of a large or medium vessel occlusion and assess the collateral supply (Fig. 1). Color-coded mCTA summation maps display all three mCTA phases in a single time-variant color map. They can be obtained from within seconds and are a useful tool which render fast and easy interpretation of mCTA images even for less experienced readers possible (Fig. 2). ${ }^{38)}$

Some people argue that more advanced imaging methods, such as CTP are beneficial since they provide additional information about the extent of the so-called ischemic "core" and the penumbra. Within 6 hours from symptom onset, however, current guidelines do not recommend advanced imaging. ${ }^{2)}$ CTP is only recommended in patients with unknown onset and those presenting $>6$ hours from onset because the late window EVT trials (DAWN and DEFUSE-3) relied mainly on CTP to determine the core-penumbra mismatch, ${ }^{39,40)}$ which was part of the trials' inclusion criteria. However, this does not mean that only late window patients in whom such a mismatch profile is present can benefit from EVT. Many late window patients are currently denied treatment because they do not meet the stringent Clinical Mismatch in the Triage of Wake Up and Late Presenting Strokes Undergoing Neurointervention With Trevo/Endovascular Therapy Following Imaging Evaluation for Ischemic Stroke 3 (DAWN/DEFUSE 3) inclusion criteria, although they could still benefit from EVT. We strongly believe that mCTA is an appropriate selection tool for early and late window patients, as it is faster, less expensive, and more robust than CTP. Of note, imaging is only one factor that determines outcome besides many others, such as patients' age, pre-morbid functional status, time from symptom onset, etc. Figure 3 outlines the management approach for EVT in AIS as it is practiced in our center. Overall, given all the data, the effectiveness of treatment and low complication rate, in our opinion, it is better to overtreat than undertreat.

\section{Workflows in the pre-EVT phase}

Although the potential for time savings is smaller in the in-hospital phase compared to the pre-hospital phase, institutional workflows should still be improved whenever possible. Some factors, such as the hospital architecture (e.g., the location of the Emergency Department, CT scanner and neuroangiography suite in relation to each other), cannot easily be solved, but they should be considered when existing hospitals are renovated or new ones are built, since a suboptimal spatial setup can lead to unnecessary treatment delays, for 


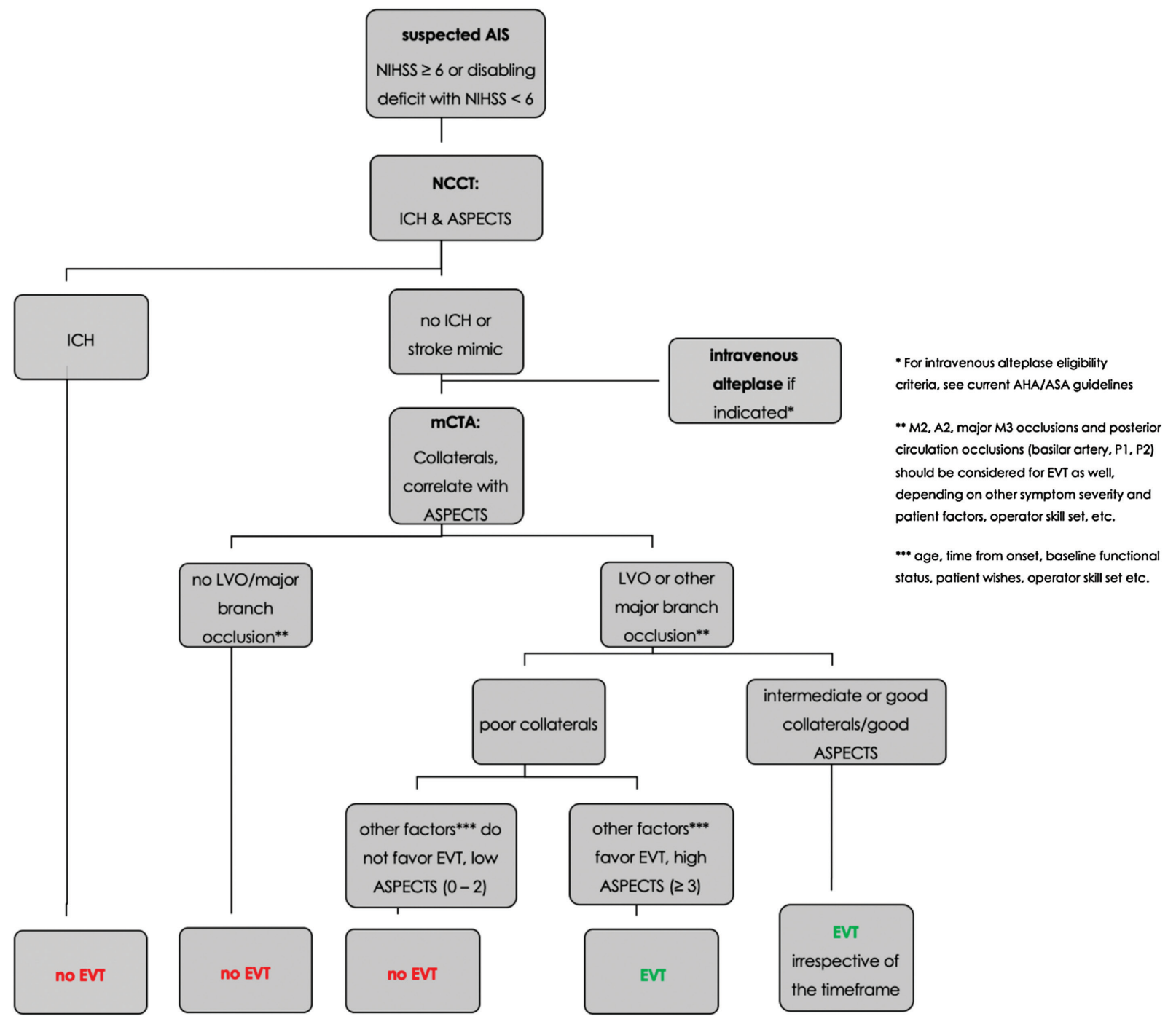

Fig. 3 Suggested management approach for endovascular treatment decision-making in acute ischemic stroke. (Reprinted with permission from: Ospel JM, Holodinsky JK, Goyal M. Management of Acute Ischemic Stroke due to Large Vessel Occlusion: JACC Focus Seminar. J Am Coll Cardiol. 2020. In press. @ American College of Cardiology Foundation)

example, due to waiting times at elevators, etc. Other workflow aspects can easily be improved upon. Table 2 lists the common problems of in-hospital workflows the pre-EVT phase and provides possible solutions to overcome these.

\section{EVT phase}

As mentioned before, fast first-pass reperfusion should be the goal when performing EVT in AIS, and this should be reflected in the thrombectomy technique as well.

The modified TICI (mTICI) scale has long been used to assess reperfusion quality after $\mathrm{EVT},{ }^{41)}$ and successful reperfusion has traditionally been defined as mTICI $2 b / 3$. However, it has been recognized that $\mathrm{mTICI} 2 \mathrm{~b}$ reperfusion yields substantially worse outcomes compared to mTICI $3.42,43)$
Hence, the expanded eTICI score has introduced a new category of "near complete" reperfusion (eTICI 2c, Table 3), and eTICI $2 c / 3$, rather than mTICI $2 b / 3$ is what we should aim for when performing EVT. The eTICI score reflects the final reperfusion result but it also matters how this final result is achieved. ${ }^{44)}$ Multiple device passes increase the risk of endothelial injury, thereby compromising procedural safety, and our goal should be to achieve complete reperfusion at the first attempt ("first-pass effect"), since this has been shown to lead to improved functional outcomes. ${ }^{18)}$

Several thrombectomy techniques are currently in use: primary aspiration (clot removal through suction), stent-retriever techniques (clot removal with a self-expanding retrievable stent), and combined approaches (e.g., CAPTIVE, ${ }^{45)}$ 
Table 2 Common problems and possible solutions of in-hospital workflows in the pre-EVT phase

\begin{tabular}{|c|c|c|c|}
\hline \multicolumn{2}{|c|}{ Problem } & Description & Solution \\
\hline \multicolumn{2}{|c|}{$\begin{array}{l}\text { Treatment delays in the } \\
\text { Emergency Department }\end{array}$} & $\begin{array}{l}\text { EVT is often delayed due to unnecessary } \\
\text { procedures in the Emergency Department } \\
\text { (ECG, chest X-rays, blood samples, patient } \\
\text { registration) }\end{array}$ & $\begin{array}{l}\text { Omitting of unnecessary steps such as } \\
\text { chest X rays, etc. } \\
\text { Patient registration as unknown } \\
\text { Bypassing the Emergency Department } \\
\text { (direct-to-CT policy/hybrid setup) }\end{array}$ \\
\hline \multicolumn{2}{|c|}{$\begin{array}{l}\text { Long preparation time to } \\
\text { arrange the equipment for } \\
\text { the EVT procedure }\end{array}$} & $\begin{array}{l}\text { Many different materials and devices are needed } \\
\text { for EVT. In the acute setting, EVT is often delayed } \\
\text { because the thrombectomy tray has to be set up, } \\
\text { appropriate syringes, catheters, etc. have to be } \\
\text { opened. }\end{array}$ & $\begin{array}{l}\text { Routine use of a pre-prepared stroke kit } \\
\text { (BRISK), }{ }^{55)} \text { which contains the basic } \\
\text { equipment that is needed for EVT }\end{array}$ \\
\hline \multicolumn{2}{|c|}{ Waiting times for anesthesia } & $\begin{array}{l}\text { Waiting for an anesthesiologist to initiate } \\
\text { general anesthesia often leads to delays in } \\
\text { EVT initiation. }{ }^{56)}\end{array}$ & $\begin{array}{l}\text { Use of conscious sedation instead of } \\
\text { general anesthesia whenever possible } \\
\text { All-time available anesthesiologist in the } \\
\text { angiography suite }\end{array}$ \\
\hline \multicolumn{4}{|c|}{ EVT: endovascular treatment } \\
\hline \multicolumn{2}{|c|}{ eTICI score } & Definition & \\
\hline 0 & \multicolumn{2}{|l|}{ No Perfusion } & \\
\hline 1 & \multicolumn{3}{|c|}{ Antegrade reperfusion past the initial occlusion, but limited distal branch filling with little or slow distal reperfusion } \\
\hline $2 a$ & \multicolumn{3}{|c|}{$\begin{array}{l}\text { Antegrade reperfusion of less than half of the occluded target artery previously ischemic territory (e.g., in one } \\
\text { major division of the MCA and its territory) }\end{array}$} \\
\hline $2 b$ & \multicolumn{3}{|c|}{$\begin{array}{l}\text { Antegrade reperfusion of more than half of the previously occluded target artery ischemic territory (e.g., in two } \\
\text { major divisions of the MCA and their territories) }\end{array}$} \\
\hline $2 c$ & \multicolumn{3}{|c|}{$\begin{array}{l}\text { Near complete perfusion except for slow flow/occlusion in a few distal cortical vessels such that at least } 90 \% \text { of } \\
\text { the territory is perfused in the parenchymal phase. }\end{array}$} \\
\hline 3 & \multicolumn{3}{|c|}{$\begin{array}{l}\text { Complete antegrade reperfusion of the previously occluded target artery ischemic territory, with absence of } \\
\text { visualized occlusion in all distal branches }\end{array}$} \\
\hline
\end{tabular}

eTICl: expanded thrombolysis in cerebral infarction score; MCA: middle cerebral artery

SAVE, ${ }^{46)}$ and BADDASS ${ }^{47)}$ ). In our opinion, when performing EVT, the BAlloon guide with large bore Distal access catheter with Dual Aspiration with Stent-retriever as Standard Approach" (BADDASS) is the optimal technique, since it combines the advantages of primary aspiration, stent retrievers, and BGC, thereby allowing for safe and efficient opening of the occluded vessel. When performing BADDASS, the stent retriever is placed in the inferior rather than the superior M2 division, since the caliber of the former one is usually larger, its course straighter and the risk of vessel injury and perforation during deployment and withdrawal of the stent therefore lower. The stent retriever is placed distally, with two-thirds of the stent beyond the clot. This ensures cell redundancy distal to the thrombus and allows us to capture distal clot fragments that might shear off during the procedure. While deploying the stent, slight forward force should be applied to ensure complete opening of the stent cells and optimal clotstent interaction. Once the stent has been deployed, the microcatheter is immediately removed out of the patient and reloaded. This saves time as a second retrieval attempt can be instantaneously initiated in case the first attempt fails. Applying slight traction to the stent wire can facilitate navigation of the distal access catheter, which should be advanced all the way to the proximal clot interface. Once the distal access catheter is in place, traction is released off the stent wire. The balloon of the guide catheter is then inflated and aspiration is applied both through the balloon guide catheter and the distal access catheter ("dual aspiration") during the clot retrieval to minimize the risk of distal embolization. Table $\mathbf{4}$ and Fig. 4 outline the key features of the BADDASS approach.

\section{Improving Stroke Care through Simulation Training}

The overall quality of stroke care depends on individual skills of the team members as well as on their ability to act 
Table 4 Key elements of the BADDASS approach ${ }^{47)}$

1 Tri-axial setup (balloon guide catheter, large bore distal access catheter, microcatheter)

2 Distal placement of a long stent retriever

3 Active push deployment

$4 \quad$ Removing and reloading the microcatheter

5 Applying traction to the stent wire

6 Releasing traction off the stent wire

7 Balloon inflation and double aspiration

8 Complete withdrawal of the stent-retriever-clot complex and distal access catheter

BADDASS: Balloon guide with large bore distal access catheter with dual aspiration with stent-retriever as standard approach

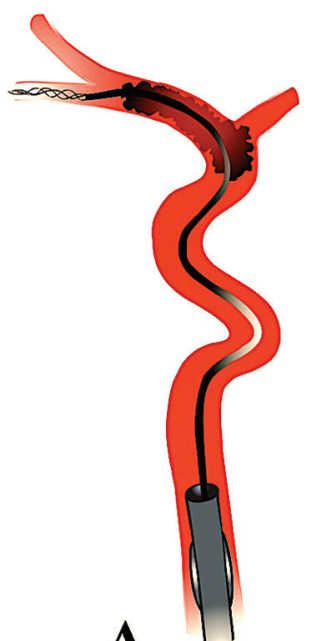

A

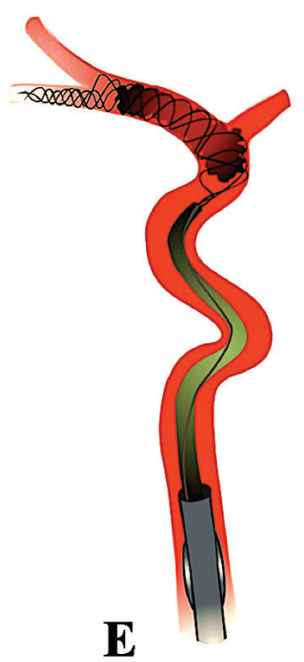

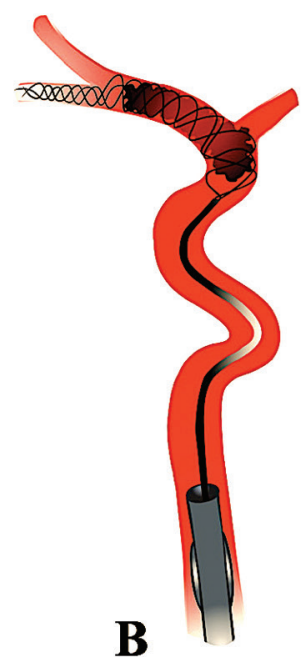

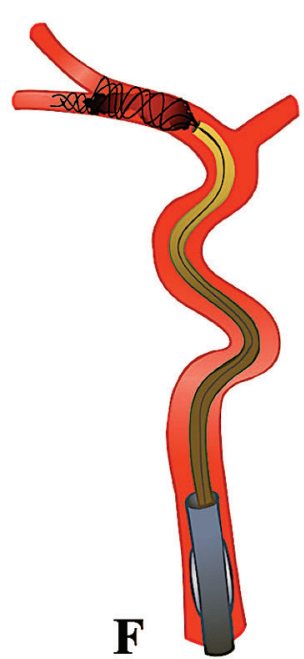

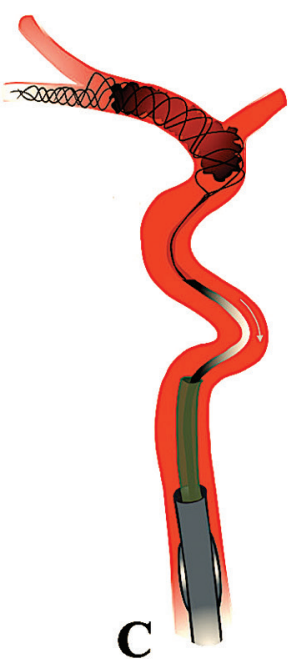
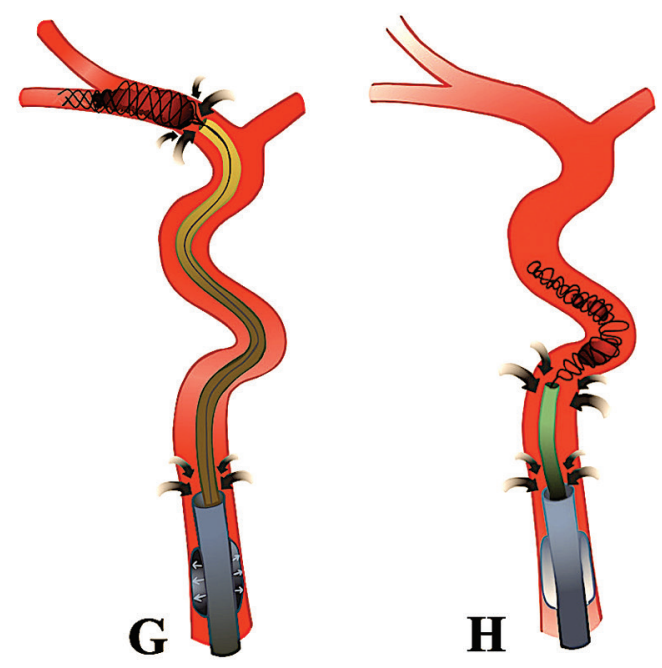

Fig. 4 Illustration of the key elements of the BADDASS approach. A balloon guide catheter is placed in the cervical internal carotid artery (A). A microwire and microcatheter, loaded with a stent retriever is then advanced past the thrombus in the inferior M2 division and the stent retriever is deployed distally (B). The microcatheter is then removed (C). Slight traction is applied to the stent wire (D) to facilitate navigation of the distal access catheter, which is advanced to the proximal clot interface (E,F). Immediately prior to clot retrieval, the balloon is inflated, aspiration is applied to the distal access catheter and the balloon guide catheter ("double aspiration" G). The distal access catheter, stent retriever, and the entrapped clot are then retrieved in the balloon guide catheter bore and removed out of the patient under maintained double aspiration (H). BADDASS: BAlloon guide with large bore Distal access catheter with Dual Aspiration with Stent-retriever as Standard Approach (Reprinted with permission from: Ospel JM, Holodinsky JK, Goyal M. Management of Acute Ischemic Stroke due to Large Vessel Occlusion: JACC Focus Seminar. J Am Coll Cardiol. 2020. In press. (c) American College of Cardiology Foundation) 
as a team. Simulator-based training can improve both these components, thereby optimizing clinical and economic outcomes in acute stroke care. The traditional teaching concept in medicine is that of graded responsibility ${ }^{48)}$ : Medical trainees who acquire their skillset while practicing on patients from the very beginning on, and with increasing experience, are granted more and more responsibility. This approach has several problems:

1) In hospitals with low stroke volumes, physicians will never be able to acquire and maintain a high level of competence.

2) Patients are frequently treated by insufficiently skilled professionals, which results in suboptimal outcomes.

3) Mistakes have often devastating consequences and are thus not openly admitted, and an unbiased analysis of medical errors does not take place.

4) The training curriculum is not standardized and depends largely on the patients who are treated during the individual physician's training period.

Simulation methods can effectively counteract these problems, since they allow physicians to acquire their skillset "ex vivo," that is, without treating patients. Simulator training has shown to improve procedural angiography times of single operators, ${ }^{49)}$ and interdisciplinary simulator-based teamtraining can lead to marked reductions in door-to-imaging times, ${ }^{50)}$ door-to-needle times, and improved patient outcomes. ${ }^{51)}$ The added value of simulation training is increasingly recognized by professional societies and incorporated in their training recommendations. ${ }^{52)}$

\section{Future Directions: Standardized Imaging Workflows, Neuroprotection and New Thrombolytic Agents}

At the time being, acute stroke imaging protocols are highly variable across different centers. This results in a lack of standardization with regard to decision-making and not infrequently leads to time delays, for example, when imaging is repeated in a drip and ship patient because the imaging protocol at the PSC where the patient was initially admitted is different from the one in the CSC. Therefore, imaging protocols as well as modality-specific thresholds (e.g., CTP core and penumbra thresholds) will have to be harmonized across vendors and software packages. Automated and standardized post-processing will reduce the degree of inter-institutional variability and ensure quick image transfer and comparability between different hospitals. Automated ASPECTS algorithms, for instance, are already reasonably accurate and will soon complement the radiologist's expert opinion. ${ }^{53)}$ Similar developments are expected for mCTA.

Pharmacological stroke treatment will dramatically change in the near future: tenecteplase, a modified tissue plasminogen activator with improved thrombolytic efficacy compared to alteplase ${ }^{54)}$ has been shown to lead to better outcomes in EVT patients and might soon complement or completely replace alteplase. Lastly, the establishment of EVT as standard of care has rendered routine fast and complete reperfusion possible. For the first time, we now have a temporary ischemia-reperfusion model in humans. This has opened the field for neuroprotective agents. Neuroprotectants increase brain cells' tolerance to temporary ischemia and help them to survive longer, until the vessel occlusion is eventually opened and reperfusion achieved. The neuroprotectant Nerinetide, for instance, can easily be administered in the pre-hospital setting and has been evaluated in acute stroke patients with LVO undergoing thrombectomy in the Safety and Efficacy of NA-1 in Subjects Undergoing Endovascular Thrombectomy for Stroke (ESCAPE NA1) trial (NCT02930018).

\section{Conclusion}

In summary, streamlining pre-hospital transfer, acute stroke imaging protocols, and in-hospital workflows as well as optimizing thrombectomy technique are essential to improve patient outcomes. Rather than over-relying on advanced imaging techniques, we should aim to stick to basic imaging for the sake of time and simplicity. Unnecessary protocols/steps/imaging/procedures prior to EVT have to be avoided. Pre-notification tools and pre-prepared stroke kits should be used so that the medical team is in an "EVT-ready state" when the patient arrives. When performing EVT, a primary combined technique such as BADDASS should be used as first-line approach, since it synergizes the advantages of stent retrievers and aspiration and thus yields the best chances to achieve fast and complete first-pass reperfusion. Standardization and automated post-processing will help to streamline the imaging protocol. Tenecteplase and neuroprotectants will probably soon change the landscape of pharmacological stroke treatment. The biggest challenge now is encountered in the pre-hospital phase, and this is where the greatest time savings can be achieved. How to get the right patient to the right hospital as fast as possible is currently the most important question that has yet to be solved. 


\section{Disclosure Statement}

Dr. Yoshimura reports honoraria from BoehringerIngelheim, Daiichi Sankyo, Bayer, Bristol-Meyers Squibb, Stryker, Medtronic, Kaneka Medics. Dr. Goyal reports licensing fees and patent royalties from GE Healthcare, Circle Neurovascular, honoraria from Stryker, Medtronic, Microvention, Mentice and research funding from Stryker and Medtronic (unrestricted research grants to the University of Calgary). Dr. Ospel reports research grants from the University of Basel Research Foundation, Julia Bangerter Rhyner Foundation and "Freiwillige Akademische Gesellschaft Basel.” Mr. Mayank has nothing to disclose.

\section{References}

1) Menon BK, Al-Ajlan FS, Najm M, et al: Association of clinical, imaging, and thrombus characteristics with recanalization of visible intracranial occlusion in patients with acute ischemic stroke. JAMA 2018; 320: 1017-1026.

2) Powers WJ, Rabinstein AA, Ackerson T, et al: 2018 Guidelines for the early management of patients with acute ischemic stroke: a guideline for healthcare professionals from the American Heart Association/American Stroke Association. Stroke 2018; 49: e46-e110.

3) Demaerschalk BM, Kleindorfer DO, Adeoye OM, et al: Scientific rationale for the inclusion and exclusion criteria for intravenous alteplase in acute ischemic stroke: a statement for healthcare professionals from the American Heart Association/American Stroke Association. Stroke 2016; 47: 581-641.

4) Goyal M, Menon BK, van Zwam WH, et al: Endovascular thrombectomy after large-vessel ischaemic stroke: a meta-analysis of individual patient data from five randomised trials. Lancet 2016; 387: 1723-1731.

5) Kunz WG, Hunink M, Almekhlafi MA, et al: Public health potential of improved reperfusion in thrombectomy for stroke based on HERMES Collaboration Data. In: 2019.

6) Saposnik G, Menon BK, Kashani N, et al: Factors associated with the decision-making on endovascular thrombectomy for the management of acute ischemic stroke. Stroke 2019; 50: 2441-2447.

7) Kashani N, Ospel JM, Menon BK, et al: Influence of guidelines in endovascular therapy decision making in acute ischemic stroke: insights from UNMASK EVT. Stroke 2019; 50: 3578-3584.

8) Wang F, Campbell BCV, Churilov L, et al: Insights into variations in preferred selection criteria for acute stroke endovascular therapy. J Neurointerv Surg 2018; 10: $542-549$.
9) Kumar P, Moy B: The cost of cancer care-balancing our duties to patients versus society: are they mutually exclusive? Oncologist 2013; 18: 347-349.

10) Pan Y, Cai X, Huo X, et al: Cost-effectiveness of mechanical thrombectomy within 6 hours of acute ischaemic stroke in China. BMJ Open 2018; 8: e018951.

11) Arora N, Makino K, Tilden D, et al: Cost-effectiveness of mechanical thrombectomy for acute ischemic stroke: an Australian payer perspective. J Med Econ 2018; 21: 799-809.

12) Achit $H$, Soudant $M$, Hosseini $K$, et al: Cost-effectiveness of thrombectomy in patients with acute ischemic stroke: the THRACE randomized controlled trial. Stroke 2017; 48: 2843-2847.

13) Shireman TI, Wang K, Saver JL, et al: Cost-effectiveness of solitaire stent retriever thrombectomy for acute ischemic stroke: results from the SWIFT-PRIME Trial (solitaire with the intention for thrombectomy as primary endovascular treatment for acute ischemic stroke). Stroke 2017; 48: 379-387.

14) Saver JL: Time is brain-quantified. Stroke 2006; 37: 263-266.

15) Menon BK, Almekhlafi MA, Pereira VM, et al: Optimal workflow and process-based performance measures for endovascular therapy in acute ischemic stroke: analysis of the Solitaire FR thrombectomy for acute revascularization study. Stroke 2014; 45: 2024-2029.

16) Liebeskind DS, Bracard S, Guillemin F, et al: eTICI reperfusion: defining success in endovascular stroke therapy. J Neurointerv Surg 2018; 11: 433-438.

17) Bourcier R, Goyal M, Liebeskind DS, et al: Association of time from stroke onset to groin puncture with quality of reperfusion after mechanical thrombectomy: a meta-analysis of individual patient data from 7 randomized clinical trials. JAMA Neurol 2019; 76: 405-411.

18) Zaidat OO, Castonguay AC, Linfante I, et al: First pass effect: a new measure for stroke thrombectomy devices. Stroke 2018; 49: 660-666.

19) Kunz W, Almekhlafi MA, Menon BK, et al: Lifetime quality of life and cost consequences of treatment delays in endovascular thrombectomy for stroke. In: 2019.

20) Froehler MT, Saver JL, Zaidat OO, et al: Interhospital transfer before thrombectomy is associated with delayed treatment and worse outcome in the STRATIS registry (systematic evaluation of patients treated with neurothrombectomy devices for acute ischemic stroke). Circulation 2017; 136: 2311-2321.

21) Lima FO, Mont'Alverne FJA, Bandeira D, et al: Pre-hospital assessment of large vessel occlusion strokes: implications for modeling and planning stroke systems of care. Front Neurol 2019; 10: 955.

22) Thorpe SG, Thibeault CM, Canac N, et al: Decision criteria for large vessel occlusion using transcranial doppler waveform morphology. Front Neurol 2018; 9: 847. 
23) Mohamad NF, Hastrup S, Rasmussen M, et al: Bypassing primary stroke centre reduces delay and improves outcomes for patients with large vessel occlusion. Eur Stroke $J$ 2016; 1: 85-92.

24) Holodinsky JK, Williamson TS, Kamal N, et al: Drip and ship versus direct to comprehensive stroke center: conditional probability modeling. Stroke 2017; 48: 233-238.

25) Schlemm E, Ebinger M, Nolte $\mathrm{CH}$, et al: Optimal transport destination for ischemic stroke patients with unknown vessel status: use of prehospital triage scores. Stroke 2017; 48: 2184-2191.

26) Calderon VJ, Kasturiarachi BM, Lin E, et al: Review of the mobile stroke unit experience worldwide. Interv Neurol 2018; 7: 347-358.

27) Wei D, Oxley TJ, Nistal DA, et al: Mobile interventional stroke teams lead to faster treatment times for thrombectomy in large vessel occlusion. Stroke 2017; 48: 3295-3300.

28) Goyal M, Jadhav AP, Wilson AT, et al: Shifting bottlenecks in acute stroke treatment. J Neurointerv Surg 2016; 8: 1099-1100.

29) Goyal M, Wilson AT, Mayank D, et al: John Nash and the organization of stroke care. AJNR Am J Neuroradiol 2018; 39: $217-218$

30) Holodinsky JK, Williamson TS, Demchuk AM, et al: Modeling stroke patient transport for all patients with suspected large-vessel occlusion. JAMA Neurol 2018; 75: $1477-1486$.

31) Lin CB, Peterson ED, Smith EE, et al: Emergency medical service hospital prenotification is associated with improved evaluation and treatment of acute ischemic stroke. Circ Cardiovasc Qual Outcomes 2012; 5: 514-522.

32) Lin CB, Peterson ED, Smith EE, et al: Patterns, predictors, variations, and temporal trends in emergency medical service hospital prenotification for acute ischemic stroke. $J \mathrm{Am}$ Heart Assoc 2012; 1: e002345.

33) Ospel JM Holodinsky JK, Goyal M: Management of acute ischemic stroke due to large-vessel occlusion. J Am Coll Cardiol 2020; 75: 1832-1843.

34) Barber PA, Demchuk AM, Zhang J, et al: Validity and reliability of a quantitative computed tomography score in predicting outcome of hyperacute stroke before thrombolytic therapy. ASPECTS Study Group. Alberta Stroke Programme Early CT Score. Lancet 2000; 355: 1670-1674.

35) Román LS, Menon BK, Blasco J, et al: Imaging features and safety and efficacy of endovascular stroke treatment: a meta-analysis of individual patient-level data. Lancet Neurol 2018; 17: 895-904.

36) Tan BY, Wan-Yee K, Paliwal P, et al: Good intracranial collaterals trump poor ASPECTS (Alberta Stroke Program Early CT Score) for intravenous thrombolysis in anterior circulation acute ischemic stroke. Stroke 2016; 47: 2292-2298.

37) Menon BK, d‘Esterre CD, Qazi EM, et al: Multiphase CT angiography: a new tool for the imaging triage of patients with acute ischemic stroke. Radiology 2015; 275: 510-520.

38) Ospel JM, Volny O, Qiu W, et al: Displaying multiphase CT angiography using a time-variant color map: practical considerations and potential applications in patients with acute stroke. AJNR Am J Neuroradiol 2020; 41: 200-205.

39) Albers GW, Marks MP, Kemp S, et al: Thrombectomy for stroke at 6 to 16 hours with selection by perfusion imaging. N Engl J Med 2018; 378: 708-718.

40) Nogueira RG, Jadhav AP, Haussen DC, et al: Thrombectomy 6 to 24 hours after stroke with a mismatch between deficit and infarct. N Engl J Med 2018; 378: 11-21.

41) Zaidat OO, Yoo AJ, Khatri P, et al: Recommendations on angiographic revascularization grading standards for acute ischemic stroke: a consensus statement. Stroke 2013; 44: 2650-2663.

42) Almekhlafi MA, Mishra S, Desai JA, et al: Not all "successful" angiographic reperfusion patients are an equal validation of a modified TICI scoring system. Interv Neuroradiol 2014; 20: 21-27.

43) Dargazanli C, Fahed R, Blanc R, et al: Modified thrombolysis in cerebral infarction $2 \mathrm{C} /$ thrombolysis in cerebral infarction 3 reperfusion should be the aim of mechanical thrombectomy: insights from the ASTER trial (contact aspiration versus stent retriever for successful revascularization). Stroke 2018; 49: 1189-1196.

44) Psychogios MN, Tsogkas I, Brehm A, et al: Clot reduction prior to embolectomy: $\mathrm{mSAVE}$ as a first-line technique for large clots. PLoS ONE 2019; 14: e0216258.

45) McTaggart RA, Tung EL, Yaghi S, et al: Continuous aspiration prior to intracranial vascular embolectomy (CAPTIVE): a technique which improves outcomes. $J$ Neurointerv Surg 2017; 9: 1154-1159.

46) Maus V, Henkel S, Riabikin A, et al: The SAVE Technique : Large-Scale Experience for Treatment of Intracranial Large Vessel Occlusions. Clin Neuroradiol 2018; 29: 669-676.

47) Ospel JM, Volny O, Jayaraman M, et al: Optimizing fast first pass complete reperfusion in acute ischemic stroke the BADDASS approach (BAlloon guiDe with large bore distal Access catheter with dual aspiration with stentretriever as standard approach). Expert Rev Med Devices 2019; 16: 955-963.

48) Pickersgill $\mathrm{T}$ : The European working time directive for doctors in training. BMJ 2001; 323: 1266.

49) Spiotta AM, Rasmussen PA, Masaryk TJ, et al: Simulated diagnostic cerebral angiography in neurosurgical training: a pilot program. J Neurointerv Surg 2013; 5: 376-381. 
50) Tahtali D, Bohmann F, Rostek P, et al: Crew resource management and simulator training in acute stroke therapy. Nervenarzt 2016; 87: 1322-1331.

51) Ajmi SC, Advani R, Fjetland L, et al: Reducing door-toneedle times in stroke thrombolysis to $13 \mathrm{~min}$ through protocol revision and simulation training: a quality improvement project in a Norwegian stroke centre. BMJ Qual Saf 2019; 28: 939-948.

52) Day AL, Siddiqui AH, Meyers PM, et al: Training standards in neuroendovascular surgery: program accreditation and practitioner certification. Stroke 2017; 48: 2318-2325.

53) Kuang H, Najm M, Chakraborty D, et al: Automated ASPECTS on noncontrast CT scans in patients with acute ischemic stroke using machine learning. AJNR Am J Neuroradiol 2019; 40: 33-38.
54) Campbell BCV, Mitchell PJ, Churilov L, et al: Tenecteplase versus alteplase before thrombectomy for ischemic stroke. N Engl J Med 2018; 378: 1573-1582.

55) Zerna $C$, Assis $Z$, d'Esterre $C D$, et al: Imaging, intervention, and workflow in acute ischemic stroke: the Calgary approach. AJNR Am J Neuroradiol 2016; 37: 978-984.

56) Menon BK, Sajobi TT, Zhang Y, et al: Analysis of workflow and time to treatment on thrombectomy outcome in the endovascular treatment for small core and proximal occlusion ischemic stroke (ESCAPE) randomized, controlled trial. Circulation 2016; 133: 2279-2286.

57) Behme D, Tsogkas I, Colla R, et al: Validation of the extended thrombolysis in cerebral infarction score in a real world cohort. PLOS ONE 2019; 14: e0210334. 